\title{
CONTRIBUIÇÃO DA RADIOGRAFIA PANORÂMICA NO DIAGNÓSTICO DA OSTEOPOROSE
}

\section{CONTRIBUTION OF THE PANORAMIC RADIOGRAPH ON THE DIAGNOSIS OF THE OSTEOPOROSIS}

\author{
Gustavo Chab Pistelli* \\ Vanessa Abreu Sanches Marques* \\ Lilian Cristina Vessoni Iwaki** \\ Mariliani Chicarelli da Silva ${ }^{* * *}$ \\ Neli Pieralissi ${ }^{* * *}$ \\ Elen de Souza Tolentino***
}

\begin{abstract}
RESUMO
Considerada como a epidemia silenciosa do século pela Organização Mundial de Saúde, a osteoporose afeta milhões de pessoas em todo o mundo. A perda de osso ocorre progressivamente e muitas vezes sem sintomas, até que uma fratura ocorra pela primeira vez. A densitometria é hoje o exame de referência para o diagnóstico da osteoporose. A osteoporose também pode apresentar manifestações na cavidade oral e, baseando-se nesses achados, alguns estudos têm procurado demonstrar que a radiografia panorâmica pode auxiliar no diagnóstico da osteoporose. Este trabalho tem como objetivo discutir a utilização da radiografia panorâmica no diagnóstico precoce da osteoporose por meio de revisão de literatura.
\end{abstract}

Descritores: Osteoporose • Radiografia Panorâmica • Diagnóstico

\section{ABSTRACT}

Considered the silent epidemic of the century by the World Health Organization, the osteoporosis affects millions of people worldwide. The bone loss occurs gradually and often without symptoms until a fracture occurs. Densitometry is today the reference test for the diagnosis of osteoporosis. Osteoporosis can also present manifestations in the oral cavity and, based on these findings, some studies have attempted to demonstrate that the panoramic radiograph can assist in the diagnosis of osteoporosis. This study aims to discuss the use of panoramic radiography in early diagnosis of osteoporosis by means of literature review.

Descriptors: Osteoporosis • Radiography, Panoramic • Diagnosis

* Aluno do Curso de Especialização em Radiologia Odontológica do Instituto Ravel de Ensino Superior (drgstv@hotmail.com).

** Aluna do curso de graduação na Universidade Estadual de Maringá, Maringá, PR, Brasil (van.marqs@gmail.com).

*** Professora da disciplina de Radiologia e Estomatologia da Universidade Estadual de Maringá (lilianiwaki@gmail.com, mchicarelli1@gmail.com, nelipieralisi@gmail.com, elen_tolentino@hotmail.com). 
PISTELLI GC

MARQUES VAS

IWAKI LCV

SILVA MC

PIERALISSI N

TOLENTINO ES

CONTRIBUIÇÃO

DA RADIOGRAFIA

PANORÂMICA NO

DIAGNÓSTICO DA

OSTEOPOROSE
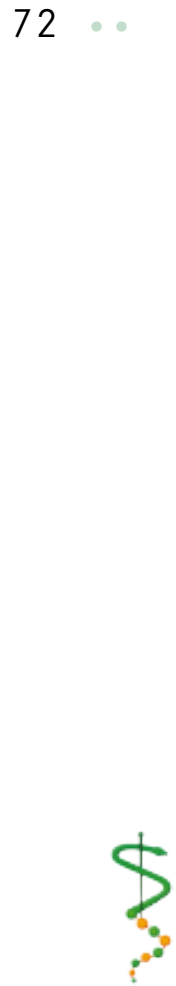

REV, ODONTOL.

UNIV, CID, SÃO

PAULO

$2014 ; 26(1): 71$ -

80 , JAN-ABR

\section{IN T RO DUÇÃO}

A Osteoporose é atualmente considerada um problema de saúde pública que afeta milhões de pessoas em todo o mundo de acordo com a International Osteoporosis Foundation (IOF) ${ }^{1}$. Considerada pela Organização Mundial de Saúde (OMS) como a epidemia silenciosa do século, a osteoporose é um distúrbio osteometabólico, na qual a densidade e qualidade óssea são reduzidas. A perda de osso ocorre progressivamente e muitas vezes sem sintomas, até que uma fratura ocorra pela primeira vez. As fraturas mais comuns associadas à osteoporose ocorrem no quadril, coluna e punho ${ }^{2}$. Segundo a IOF, uma em cada três mulheres com mais de 50 anos sofrerão fraturas causadas pela osteoporose, assim como um em cada cinco homens ${ }^{1}$.

De acordo com a OMS, a osteoporose atinge mais de 75 milhões de pessoas na Europa, Japão e EUA, causando mais de 2,3 milhões de fraturas anualmente na Europa e EUA. Na Europa, a cada 30 segundos acontece uma fratura por osteoporose e no mundo todo estima-se uma a cada 3 segundos. Nos Estados Unidos da América ocorrem cerca de 1,5 milhões de fraturas por $\mathrm{ano}^{2}$.

A Sociedade Brasileira de Osteoporose (SOBRAO) estima, considerando o último censo do IBGE, que existam 5,5 milhões de brasileiros com osteoporose e a IOF 10 milhões ${ }^{1}$. Segundo dados fornecidos pelo Ministério da Saúde, em 2009, foram gastos R \$ 57,61 milhões com internações (até outubro) e R $\$ 24,77$ milhões com medicamentos para tratamento da osteoporose ${ }^{3}$.

Homens e mulheres com mais de 60 anos de idade correm maior risco de osteoporose do que os mais jovens. No entanto, é possível ter osteopenia (baixa massa óssea) ou osteoporose muito mais cedo. Os principais tipos de osteoporose são: a osteoporose pós-menopausa, que como o próprio nome diz acomete as muIheres após a menopausa, a osteoporose senil que atinge pessoas com mais de 70 anos e a osteoporose secundária atingindo pessoas com doença renal, hepática, endócrina, hematológica ou que usam alguns medicamentos, como, por exemplo, corticoides ${ }^{1}$.

A densitometria é hoje o exame de referência para o diagnóstico da osteoporose. É realizada por técnica de DXA - absorciometria por raios $X$ com dupla energia de baixa radiação - e fornece valores reprodutíveis em sítios importantes de fraturas associadas à osteoporose. Os valores obtidos por meio da densitometria são comparados a valores médios de adultos jovens, considerados os valores de referência (T-score). Esses valores representam o pico da massa óssea e são apresentados em $\mathrm{g} / \mathrm{cm}^{2}(1,31)$. Os valores de referência da densidade mineral óssea (DMO) considerada normal são: na coluna lombar igual ou maior que $1,05 \mathrm{~g} / \mathrm{cm}^{2}$ e igual ou maior que $0,83 \mathrm{~g} / \mathrm{cm}^{2}$ no colo do fêmur ${ }^{4}$.

Em 1994, a OMS definiu os critérios atualmente utilizados nos laudos de densitometria óssea em todo o mundo. Os critérios são os seguintes: a) normal: desvio-padrão de até $-1,00$; b) osteopenia: desvio-padrão compreendido entre $-1,00$ e $-2,50$; c) osteoporose: desvio-padrão menor ou igual a $-2,50^{5}$.

As radiografias panorâmicas (RPs) estão sendo cada vez mais utilizadas como diagnóstico complementar das doenças bucais. Atualmente, muitos estudos vêm sendo realizados com o intuito de usar essas radiografias para o diagnóstico da osteoporose, uma vez que alguns autores associam a osteoporose com alterações no trabeculado mandibular.

A espessura da cortical mandibular (ECM), assim como sua morfologia (MCM) podem ser utilizadas para avaliação da DMO da mulher6, 7. Dessa forma, esses exames radiográficos podem ser utilizados como contribuição no diagnóstico da doença, possibilitando o encaminhamento mais precoce para o tratamento adequado.

O objetivo desse trabalho é discutir, por meio de revisão de literatura, se alterações radiográficas visualizadas em radiografias panorâmicas podem ser usadas como ferramenta de diagnóstico para diferenciar pacientes normais de pacientes osteoporóticos.

\section{DESEN V OLVIMENTO}

A osteoporose, que literalmente significa "osso poroso", é uma doença na qual 
a densidade e qualidade óssea são reduzidas. Como os ossos se tornam mais porosos e frágeis, o risco de fratura torna-se muito maior ${ }^{1}$.

A perda de osso ocorre silenciosa e progressivamente. Muitas vezes, não há sintomas até que a fratura ocorra pela primeira vez. É também conhecida como epidemia silenciosa por causa de seu desenvolvimento assintomático e da falta de conscientização pública. A osteoporose foi considerada a epidemia silenciosa do século pela OMS. As fraturas mais comuns associadas à osteoporose ocorrem no quadril, coluna e punho ${ }^{2}$.

Se os resultados de um teste de DMO forem positivos para osteopenia ou osteoporose, isso não significa automaticamente que o paciente terá uma fratura. Algumas mudanças no estilo de vida e uma série de terapias disponíveis podem retardar a perda óssea e ajudar a prevenir fraturas ${ }^{3}$.

A osteoporose também pode apresentar manifestações na cavidade oral, caracterizadas por: reabsorção do processo alveolar, perda dentária, doença periodontal crônica destrutiva, dores relacionadas ao seio maxilar ou fraturas ${ }^{8}$. As porções trabecular e cortical respondem de maneira diferente à reabsorção ${ }^{9}$.

Groen, Duyvensz e Halsted $^{10}$, em 1960, foram os primeiros pesquisadores a associar a osteoporose à perda óssea na mandíbula e na maxila dos seres huma$\operatorname{nos}^{10}$.

Em 1974, Wical e Swoope ${ }^{11}$ realizaram medições em 260 radiografias panorâmi-

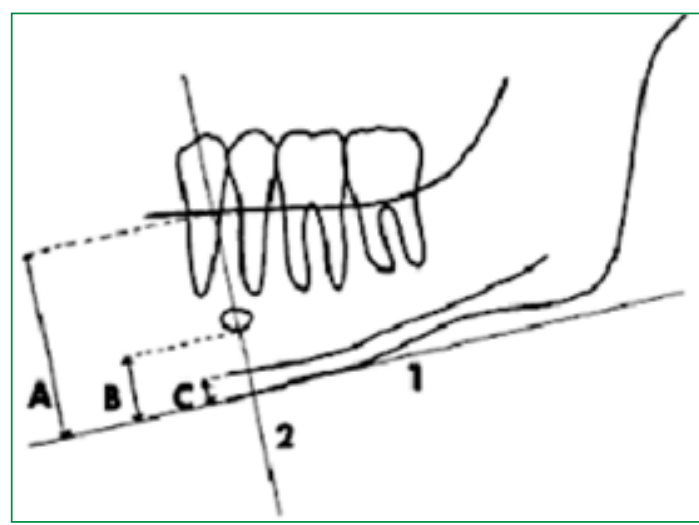

Figura 1 - Desenho esquemático das linhas e medidas realizadas em radiografias panorâmicas ${ }^{11}$. cas de mandíbulas normais. Eles observaram que numa elevada percentagem de filmes, independentemente da ampliação ou da distorção habitual das imagens, a borda inferior do forame mentual estava muito próxima de uma linha que divide a mandíbula em três partes. Para os autores, em uma mandíbula não reabsorvida, a distância da base da mandíbula à margem inferior do forame mentual é de cerca de 1/3 da altura total da mandíbula na mesma região (Figura $2 \mathrm{~A}$ ). Os autores se basearam no fato de que a relação entre o forame mentual e a borda inferior da mandíbula permanece relativamente constante ao longo da vida ${ }^{11}$.

Primeiro se marcava um traço na margem inferior do forame mentual; em seguida, se desenhava uma linha paralela ao longo do eixo da mandíbula, tangencial à sua borda inferior; por último, uma linha perpendicular a essa tangente, que fazia interseção com a borda inferior do forame mentual (Figura 2). A partir daí, algumas medidas foram realizadas: A, altura total da mandíbula (altura do rebordo residual): distância da borda inferior da mandíbula ao topo da crista alveolar; B, altura basal: distância da borda inferior da mandíbula à margem inferior do forame mentual; C, espessura da cortical mandibular (Figura 1). Assim, os autores calcularam a percentagem de perda óssea mandibular por meio

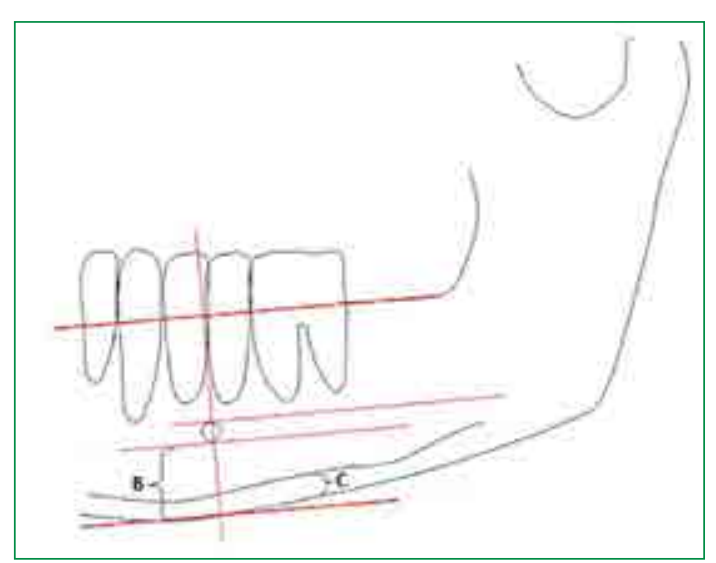

Figura 2 - As medidas são definidas como: $C$ - espessura da cortical, B - limite inferior do canal mandibular à base da mandibula. $I P M=C / B^{8,17}$.
PISTELLI GC MARQUES VAS IWAKI LCV

SILVA MC

PIERALISSI N

TOLENTINO ES

CONTRIBUIÇÃO DA RADIOGRAFIA PANORÂMICA NO DIAGNÓSTICO DA OSTEOPOROSE

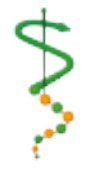

REV, ODONTOL. UNIV, CID, SÃO PAULO 2014; 26(1): 71 80 , JAN-ABR 
PISTELLI GC

MARQUES VAS

IWAKI LCV

SILVA MC

PIERALISSI N

TOLENTINO ES

CONTRIBUIÇÃO

DA RADIOGRAFIA

PANORÂMICA NO

DIAGNÓSTICO DA

OSTEOPOROSE

74
I SSN 1983-5183

da equação 3B-A (Figura 1), sendo 3B a altura inicial da mandíbula e A, a distância entre os bordos inferior e superior da mandíbula reabsorvida (Figura $2 \mathrm{~A})^{11}$.

Os autores sugerem que este método de estimar e descrever o grau de reabsorção do osso pode facilitar o estudo da osteoporose proporcionando uma base anatômica para classificar ou agrupar os pacientes afetados, em vários graus da doença ${ }^{11}$.

Em 1991, Benson, Prihoda e Glass ${ }^{8}$ realizaram um estudo no qual observaram e avaliaram diferenças nos índices em uma população de 353 indivíduos adultos, divididos igualmente por sexo, idade (30 a 79 anos) e grupo racial (negros, hispânicos, brancos). Os dados foram analisados com respeito a lados (direito e esquerdo), a raça, sexo, idade e combinações dessas variáveis ${ }^{8}$.

Com base na técnica de Wical e Swoope ${ }^{11}$, Benson et al. ${ }^{8}$ (1991) definiram um novo índice radiomorfométrico de massa óssea mandibular cortical, o Índice Panorâmico Mandibular (IPM), que utilizava as mesmas medidas anteriores A, B C (Figura 1). Esse índice é representado pela razão entre a espessura da cortical mandibular (C) e a distância entre o limite inferior do forame mentual e a base da mandíbula (B), cujo valor considerado normal é $\geq$ a 0,3 (Figura 2). A distância da margem inferior do forame mentual à borda inferior da mandíbula foi determinada como padrão devido à dificuldade em identificar a margem superior do mesmo, onde os processos alveolares foram severamente reabsorvidos ${ }^{8}$.

Os autores observaram o IPM em uma população de 353 indivíduos adultos, divididos igualmente por gênero, idade (30 a 79 anos) e grupo racial (negros, hispânicos, brancos). Os resultados demonstraram que negros tiveram um IPM de maior média do que hispânicos ou brancos, que eram estatisticamente semelhantes. Mudanças relacionadas à idade, comparando-se os grupos etários mais jovens e mais velhos, dentro de cada sexo e grupo racial, indicaram uma diminuição significativa no IPM médio com o aumento da idade em mulheres negras e hispânicas ${ }^{8}$.

Klemetti, Kolmakov e Kröger ${ }^{6}$, em 1994, introduziram a classificação morfológica da cortical mandibular (IMC) por meio da observação da região posterior ao forame mentual, classificando-a em 3 grupos: C1 (córtex normal): a margem endosteal do córtex está nítida, afilada em ambos os lados; C2 (córtex com erosão suave a moderada): a margem endosteal mostra defeitos semilunares (reabsorção
REV, ODONTOL. UNIV. CID. SÃO PAULO $2014 ; 26(1): 71-$ $80, J A N-A B R$

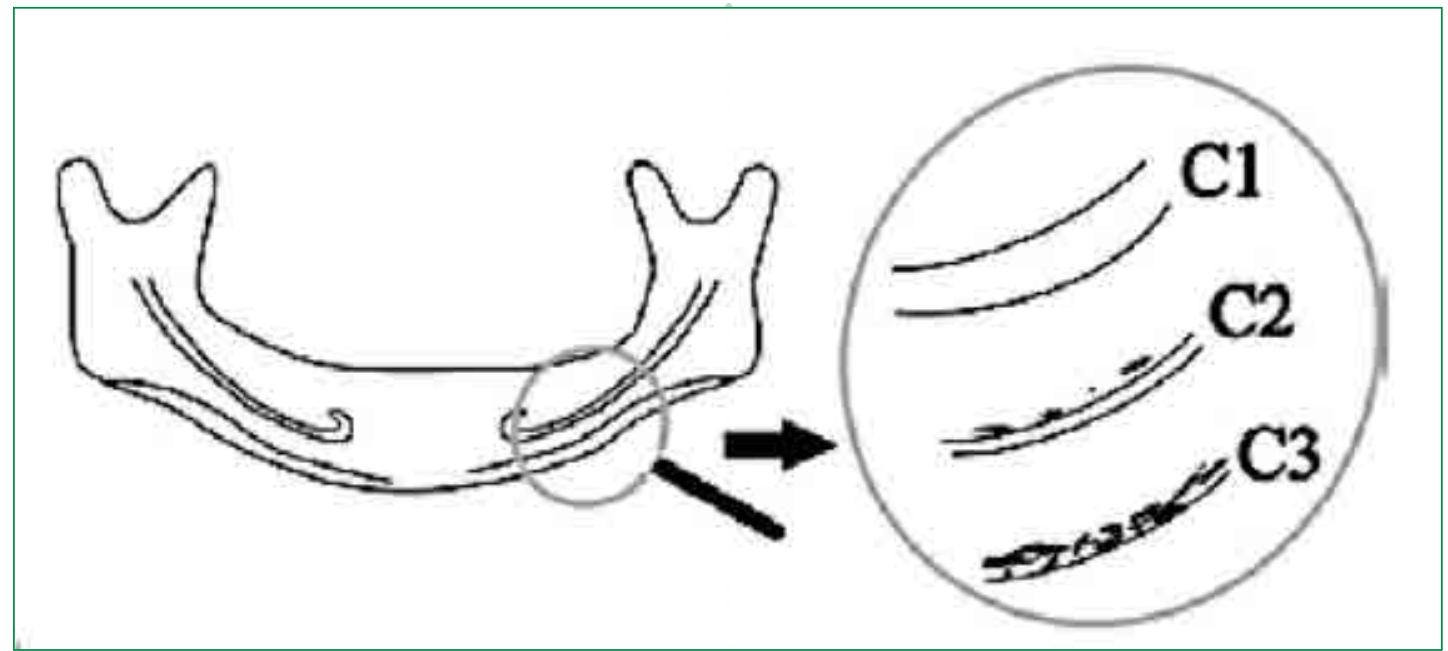

Figura 3 - Índice Mandibular cortical adaptado por Klemetti, Kolmakov e Kroger ${ }^{6}$. 
em lacunas) ou resíduos de cortical endosteal (uma a três camadas) e C3 (córtex com erosão severa): a camada cortical está claramente porosa e com espessura reduzida e possui resíduos endosteais corticais densos (Figura 3).Os autores afirmam que, quando a altura da cortical for menor que $4 \mathrm{~mm}$ e classificada como C3, o paciente é de risco para o desenvolvimento da osteoporose. Porém quando a altura da cortical for alta e classificada como C1, trata-se de um paciente de baixo risco $^{6}$.

Em 1996 Law, Bollen e Chen ${ }^{12}$ realizaram um estudo com radiografias periapicais, oclusais e panorâmicas de 111 mulheres adultas com presença de dentes. Para serem usadas nesse estudo, as radiografias deveriam mostrar a presença de um pré-molar e um molar adjacentes em pelo menos um dos lados da mandíbula. Esse estudo comparou quatro métodos de estimativa de densidade óssea de radiografias odontológicas: um contra o outro e contra a tomografia computadorizada quantitativa e absorciometria simples e dupla de raios X (DXA e SXA). Os autores concluíram que um método por si só não pode ser usado para diagnosticar a osteoporose. A análise discriminante feita nesse estudo mostra que uma combinação das análises de dimensão fractal, microdensitometria e intensidade de pixel classificaram $83 \%$ dos participantes corretamente, enquanto uma combinação da tomografia quantitativa computadorizada, absorciometria simples de fóton e a absorciometria dupla de fótons classificaram $78 \%$ dos participantes corretamente ${ }^{12}$.

Tagushi et al. ${ }^{7}$ (1996) realizaram um estudo que consistia em avaliar as medidas da forma e da largura da cortical inferior de mandíbulas de mulheres pós-menopáusicas, por meio de radiografias panorâmicas, buscando esclarecer se tais medidas são validadas em comparação com ferramentas simples de triagem com base em questionários, como a ferramenta de autoavaliação da osteoporose e se essas medidas podem ser utilizadas em muIheres na pós-menopausa com história de histerectomia, ooforectomia ou o uso de estrogênio. Foram utilizadas 200 radiografias de mulheres que não deveriam ter nenhuma doença óssea metabólica, câncer com metástase óssea, insuficiência renal grave ou que fizessem uso de medicamentos que afetassem o metabolismo ósseo. Concluiu-se que dentistas podem ser capazes de encaminhar as mulheres na pós-menopausa com suspeita de osteoporose na coluna com base em radiografias panorâmicas, com desempenho diagnóstico semelhante ao das ferramentas de rastreio de osteoporose realizado através de questionários ${ }^{7}$.

Ledgerton et al. ${ }^{13}$ em 1999 realizaram um estudo com 500 mulheres que haviam sido submetidas a exame radiográfico panorâmico como parte de seu tratamento dentário. As pacientes foram agrupadas de acordo com a idade. O grupo mais jovem foi de 25 a 29 anos e o mais velho 70 a 74 anos. Cinco índices foram medidos bilateralmente nas radiografias panorâmicas: largura da cortical no gônio (Gl) e abaixo do forame mentual (IM), o índice panorâmico mandibular (IPM), o índice mandibular cortical (IMC) e a medida da largura cortical no antegônio (Al). As medidas foram analisadas para facilitar a aplicação, reprodutibilidade, as relações com a idade, dentição, classe social e inter-relações entre as variáveis. Os autores concluíram que, apesar de inicialmente todos os índices pareceram de fácil aplicação, todos eles demonstraram algumas limitações nas medidas que afetaram a reprodutibilidade. Isso foi particularmente verdadeiro para as medições interobservador no estudo-piloto. No entanto, as medições repetidas intraobservador não demonstraram diferenças significativas e a precisão foi moderada para todos os índices lineares exceto GI, enquanto IMC teve excelente reprodutibilidade. Todos os índices demonstraram uma correlação negativa com a idade e uma diferença significativa entre as mulheres mais jovens e as mais velhas ${ }^{13}$.

Lee et al. ${ }^{14}$, em 2005, analisaram radiografias panorâmicas e densitometrias ósseas da coluna e do colo femoral em 100 mulheres entre 50 e 84 anos. As radiografias panorâmicas foram avaliadas
PISTELLI GC MARQUES VAS IWAKI LCV

SILVA MC

PIERALISSI N

TOLENTINO ES

CONTRIBUIÇÃO

DA RADIOGRAFIA

PANORAMICA NO

DIAGNÓSTICO DA

OSTEOPOROSE

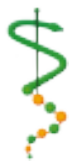

REV, ODONTOL.

UNIV. CID, SÃo

PAULO

2014; 26(1): 71 -

80 , JAN-ABR 


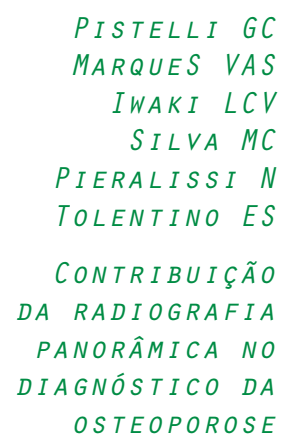

76

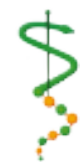

REV. ODONTOL.

UNIV. CID. SÃO

PAULO

$2014 ; 26(1): 71$

80, JAN-ABR

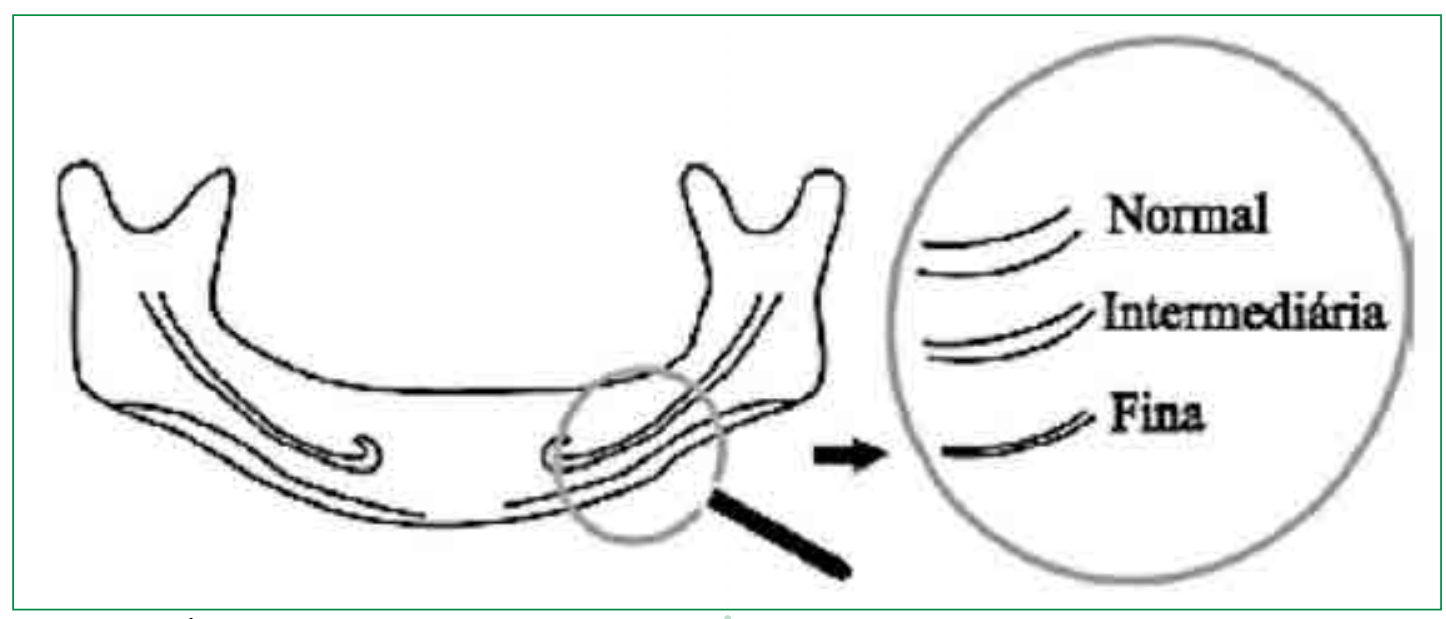

Figura 4 - Índice visual modificado proposto por Leite ${ }^{15}$.

por quatro cirurgiões-dentistas, dos quais três eram especialistas em radiologia. Considerando que a simples estimativa visual da espessura da cortical mandibular em radiografias panorâmicas poderia ser ferramenta auxiliar na identificação de mulheres na pós-menopausa com baixa DMO, os autores classificaram a cortical em fina e não fina. Não houve diferença entre a estimativa visual e a mensuração da cortical. A sensibilidade, a especificidade e o valor preditivo positivo encontrados foram de $57,7 \%$, $82 \%$ e $72,5 \%$, respectivamente ${ }^{14}$.

Leite $^{15}$, no ano de 2008, observou, em um estudo com 351 mulheres na pós-menopausa, que uma simples estimativa visual da cortical da mandíbula, classificando-a em espessura normal, intermediária e muito fina (Figura 4), poderia predizer o diagnóstico de osteoporose de T-score $\leq 2,0$. Esse índice foi nomeado de visual modificado por se tratar de uma modificação baseada na classificação de Lee et al. ${ }^{14}, 2005$.

O autor também verificou uma correlação entre os índices mandibular cortical, visual modificado, mentual e as DMOs da coluna lombar, do colo femoral e do fêmur total. As mulheres classificadas como C3, pelo índice mandibular cortical, possuem razões de chances de 5,16; 3,35 e 4,57 para o diagnóstico densitométrico de osteoporose da coluna lombar em L1-L4 eT-score $\leq-2,0$ em L1-L4 e no fêmur proximal, respectivamente. As mulheres na pós-menopausa com afilamento acentuado da cortical mandibular, avaliado pelo índice visual modificado, possuem razões de chances de 15,$13 ; 5,21$ e 10,11 respectivamente, para o diagnóstico densitométrico de osteoporose em L1-L4, no fêmur proximal e para identificar $\mathrm{T}$-score $\leq-2,0$ em L1-L4 ${ }^{15}$.

Em 2008 Mahl et al. ${ }^{16}$ realizaram um estudo constituído por amostra de mulheres com mais de 40 anos que foram encaminhadas para a realização de radiografia panorâmica para tratamento odontológico. As pacientes deveriam ter realizado exame de densitometria óssea em um período não superior a três meses antes da panorâmica, e deveriam seguir os seguintes critérios de inclusão: pós-menopausa, não estarem usando terapia de reposição hormonal ou de cálcio, não terem sofrido histerectomia ou ooforectomia e não apresentarem doenças metabólicas relacionadas à osteoporose. Além disso, foram excluídas da amostra mulheres em que se constatou presença de lesão óssea, fratura, deformidade ou cirurgia prévia na mandíbula. As radiografias foram mensuradas de forma a se obterem os seguintes índices radiomorfométricos: índice panorâmico mandibular (IPM), índice mentoniano (IM), índice antegonial (IA), índice goníaco (IG) e índice cortical mandibular (ICM). Os dados foram organizados para comparação com os resultados da densitometria óssea e os autores concluíram que os índices avaliados foram reprodutíveis. Os índices panorâmico mandibular e mentual foram os que apresentaram maiores valores de sensibilidade para detectar osteopenia/osteoporose, porém a especificidade do índice panorâmico mandibular foi baixa; todos os índices avaliados foram 
capazes de identificar baixa massa óssea, contudo, apenas os índices panorâmico mandibular e mentual permitiram diferenciar pacientes com osteopenia/osteoporo$\mathrm{se}^{16}$.

Em 2011, López et al. ${ }^{17}$ propuseram validar um sistema assistido por computador para medir três diferentes índices mandibulares: largura cortical, índice panorâmico mandibular e índice de reabsorção óssea alveolar mandibular. Realizaram o estudo no qual 10 examinadores diferentes selecionaram aleatoriamente 10 radiografias panorâmicas de 10 pacientes, a partir de um conjunto de 200 radiografias. O resultado foi que o sistema computadorizado proposto demonstra taxas superiores de reprodutibilidade em relação aos métodos manuais. Além disso, o tempo necessário para realizar as medições utilizando o método proposto é insignificante em comparação com a realização das medidas manualmente. A conclusão do estudo foi que o sistema fornece uma forma prática para executar essas medidas, não requer um examinador especialista e não leva mais do que 16 segundos por análise. Assim, pode ser adequado para o diagnóstico da osteoporose usando radiografias panorâmicas ${ }^{17}$.

Kavita et al. ${ }^{18}$, em 2012, realizaram um estudo com 40 mulheres para avaliar a eficácia de um mecanismo de suporte vetorial (SVM) na análise da largura da cortical mandibular em radiografias panorâmicas, a fim de identificarem mulheres na pósmenopausa com baixa densidade óssea, podendo reduzir diagnósticos equivocados. A sensibilidade e a especificidade para identificar mulheres com baixa DMO em qualquer região da coluna lombar ou colo do fêmur foram $90,6 \%$ e $80,9 \%$ respectivamente. Os resultados sugerem que o sistema recentemente desenvolvido com o método SVM seria útil para identificar mulheres pós-menopáusicas com DMO esquelética baixa ${ }^{18}$.

Atualmente, os pacientes odontológicos estão sendo encaminhados com mais frequência para realização de exames radiográficos panorâmicos, visto que esse método de auxílio diagnóstico é mais acessível e capaz de detectar as alterações morfológicas da mandíbula decor- rentes da idade.

De acordo com os estudos de Wical e Swoope ${ }^{11} 1974$, assim como os de Benson, Prihoda e Glass ${ }^{8} 1991$, estimar e descrever o grau de reabsorção do osso alveolar em radiografias panorâmicas podem auxiliar no diagnóstico e estudo da osteoporose. Esses estudos mostram que existem diferenças significativas nas medidas realizadas na mandíbula em radiografias panorâmicas de pacientes portadores de osteoporose. No entanto, os índices propostos pelos autores podem apresentar algumas dificuldades na sua identificação, como o reconhecimento pelos examinadores do forame mentual nas radiografias, assim como variações no tamanho, posição e aparência radiográfica do forame mentual.

Para Klemetti et al. ${ }^{6}$ 1994, indivíduos com osteoporose estão mais sujeitos a apresentar erosões na cortical inferior da mandíbula do que o grupo-controle, e a espessura dessa cortical é reduzida em indivíduos com osteoporose. Lee et al. ${ }^{14}$ 2005 e Leite ${ }^{15}$ 2008, concordam que, em uma simples observação visual da espessura da cortical inferior da mandíbula, é possível determinar a presença ou não de osteoporose. Deve ser ressaltado que Lee et al. ${ }^{14} 2005$, não consideraram a existência de estágio intermediário que pode ser correlacionado ao diagnóstico densitométrico de osteopenia, do mesmo modo que o estágio C2 do índice mandibular cortical.

Alguns estudos ${ }^{8,16}$ mostram que existe diferença significativa na medida do IPM entre os três grupos de pacientes pós-menopausa (normal, osteopenia e osteoporose), o que caracteriza a radiografia panorâmica como uma ferramenta válida no diagnóstico da osteoporose.

A vantagem da utilização de radiografias panorâmicas é que, muitas vezes, elas já estão disponíveis e, assim, o paciente não tem de ser exposto à radiação adicional para diagnosticar a osteoporose ${ }^{12}$. Ledgerton et al. ${ }^{13} 1999$, propuseram um método computadorizado para as mensurações das radiografias panorâmicas. Os custos de um software e um computador para essas avaliações ainda são pequenos quando comparados ao equipamento-pa-
PISTELLI GC MARQUES VAS IWAKI LCV

SILVA MC

PIERALISSI N

TOLENTINO ES

CONTRIBUIÇÃO

DA RADIOGRAFIA

PANORAMICA NO

DIAGNÓSTICO DA

OSTEOPOROSE
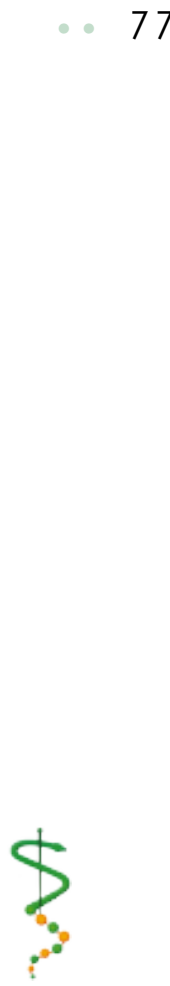

REV, ODONTOL.

UNIV. CID, SÃo

PAULO

2014; 26(1): 71 .

80 , JAN-ABR 
PISTELLI GC

MARQUES VAS

IWAKI LCV

SILVA MC

PIERALISSI N

TOLENTINO ES

CONTRIBUIÇÃO

DA RADIOGRAFIA

PANORÂMICA NO

DIAGNÓSTICO DA

OSTEOPOROSE

78

\section{SSN 1983-5183}

drão para diagnóstico da osteoporose. Se os índices realizados na mandíbula são realmente precisos para o diagnóstico da osteoporose, os custos para o paciente seriam bastante reduzidos, o que representaria uma vantagem ${ }^{13}$.

Um estudo de Shintaku et al. ${ }^{19}$ 2013, investigou a possibilidade de ensinar estudantes de Odontologia a detectarem alterações radiográficas sugestivas de osteoporose. Foram selecionadas vinte e cinco radiografias panorâmicas de pacientes da própria faculdade com histórico de osteoporose e no radiográfico apresentavam alterações sugestivas da doença. Outras vinte e cinco radiografias panorâmicas normais foram selecionadas por um médico da base de dados da Faculdade de Odontologia da Universidade de Tennessee Health Science Center. Vinte estudantes foram ensinados a utilizar o índice cortical mandibular (ICM) e detectar alterações sugestivas de osteoporose. Os alunos também usaram uma escala de cinco pontos para determinar a precisão do diagnóstico de imagens panorâmicas para a osteoporose. Foram utilizados coeficiente de correlação intraclasse (CCIC) e testes estatísticos de coeficientes internos de Cronbach alfa para avaliar a confiabilidade dos examinadores entre os vinte alunos e entre os alunos e o radiologista. Para testar as diferenças de diagnóstico entre o padrão-ouro (clínico dental) e o radiologista, foi realizado o teste McNemardo qui-quadrado para dados emparelhados. A consistência entre examinadores foi excelente, tanto para os alunos $(\alpha=0,902)$ como entre os alunos e o radiologista $(\alpha$ $=0,909)$. A precisão do diagnóstico de imagens panorâmicas foi moderada $(A z$
$=0,81$. Não houve diferença estatisticamente significativa entre as avaliações radiográficas e clínicas $(p=0,0801$; McNemar do qui-quadrado $=3,063)$. Ensinar estudantes de Odontologia a reconhecerem alterações radiológicas sugestivas de osteoporose em radiografias panorâmicas de rotina deve ser enfatizado para melhorar a sua consciência e identificação da doença.

Entretanto, acreditamos que os estudos utilizando radiografias panorâmicas ainda não são absolutamente confiáveis. Alguns autores não encontraram uma correlação positiva entre o IPM e o teste de DMO em mulheres pós-menopáusicas ${ }^{10,13}$. Parece lógico que mulheres (e homens) acima de 50 anos apresentem uma redução da cortical mandibular devida à reabsorção óssea fisiológica comum com o avanço da idade e a perda dentária. Predizer se esses índices propostos podem realmente diagnosticar a osteoporose nos parece imprudente. Fica evidente que mais estudos são necessários.

\section{CONSIDERAÇÕES FINAIS}

Os diversos estudos demonstram que a radiografia panorâmica é uma ferramenta para diagnosticar uma baixa densidade óssea. Entretanto, seu uso para esse propósito não deve ser indiscriminado, uma vez que sua precisão para o diagnóstico da osteoporose ainda não é totalmente confiável.

\section{CONFLITO DE INTERESSES}

Os autores declaram não haver conflito de interesse que poderia se constituir em um impedimento para a publicação.

\footnotetext{
REV. ODONTOL. UNIV. CID. SÃO PAULO
} $2014 ; 26(1): 71$ 80 , JAN-ABR 
1. International Osteoporosis Foundation. Diagnosing osteoporosis. s.d. [Acesso em 201210 agosto]; Disponível em: http://www.iofbonehealth.org/ diagnosing-osteoporosis.

2. Pinto Neto Aarão Mendes. Consenso brasileiro de osteoporose. 2011 [Acesso em 201213 agosto]; Disponível em: http://ebookbrowse.com/ consenso-brasileiro-osteoporose-pdf-d49374362.

3. Portal da Sáude SUS. [Acesso em 201229 setembro ]; Disponível em http://portal.saude.gov. br/portal/saude/visualizar_texto. $c f m$ ?idtxt=33674\&janela=1.

4. Souza MPG. Diagnóstico e tratamento da osteoporose. Revista Brasileira de Ortopedia 2010 maio/jun;45(3):2209.

5. Sampaio Netto O, Coutinho LdOL, Souza DC. Análise da nova classificação de laudos de densitometria óssea. Radiologia Brasileira 2007 jan/ fev;40(1):23-5.

6. Klemetti E, Kolmakov S, Kroger $\mathrm{H}$. Pantomography in assessment of the osteoporosis risk group. Scand J Dent Res 1994 Feb;102(1):68-72.

7. Taguchi A, Suei Y, Ohtsuka M, Otani K, Tanimoto K, Ohtaki M. Usefulness of panoramic radiography in the diagnosis of postmenopausal osteoporosis in women. Width and morphology of inferior cortex of the mandible. Dentomaxillofac Radiol 1996 Nov;25(5):263-7.

8. Benson BW, Prihoda TJ, Glass BJ. Variations in adult cortical bone mass as measured by a panoramic mandibular index. Oral Surg Oral Med Oral Pathol 1991 Mar;71(3):349-56.

9. Klemetti E, Kolmakov S, Heiskanen P, Vainio P, Lassila V. Panoramic mandibular index and bone mineral densities in postmenopausal women. Oral Surg Oral Med Oral Pathol 1993 Jun;75(6):774-9.
10. Groen JJ, Duyvensz F, Halsted JA. Diffuse alveolar atrophy of the jaw (non-inflammatory form of paradental disease) and pre-senile osteoporosis. Gerontol Clin (Basel) 1960 2:68-86.

11. Wical KE, Swoope CC. Studies of residual ridge resorption. I. Use of panoramic radiographs for evaluation and classification of mandibular resorption. J Prosthet Dent 1974 Jul;32(1):712.

12. Law AN, Bollen AM, Chen SK. Detecting osteoporosis using dental radiographs: a comparison of four methods. J Am Dent Assoc 1996 Dec;127(12):1734-42.

13. Ledgerton D, Horner K, Devlin H, Worthington $\mathrm{H}$. Radiomorphometric indices of the mandible in a British female population. Dentomaxillofac Radiol 1999 May;28(3):173-81.

14. Lee K, Taguchi A, Ishii K, Suei Y, Fujita M, Nakamoto $T$, et al. Visual assessment of the mandibular cortex on panoramic radiographs to identify postmenopausal women with low bone mineral densities. Oral Surg Oral Med Oral Pathol Oral Radiol Endod 2005 100(2):226-31.

15. Leite AF, Figueiredo PTdS, Guia CM, Melo NS, Paula APd. Radiografia panorâmica: instrumento auxiliar no diagnóstico da osteoporose. Revista Brasileira de Reumatologia 2008 jul/ ago;48(4):226-33.

16. Mahl CRW, Licks R, Fontanella VRC. Comparação de índices morfométricos obtidos na radiografia odontológica panorâmica na identificação de indivíduos com osteoporose/osteopenia. Radiologia Brasileira 2008 maio/ jun;41(3):183-7.

17. Lopez-Lopez J, Alvarez-Lopez JM, Jane-Salas E, Estrugo-Devesa A, Ayuso-Montero R, Velasco-Ortega $\mathrm{E}$, et al. Computer-aided system for morphometric mandibular index computation. (Using dental panoramic radiographs). Med Oral Patol Oral Cir Bucal 2012 Jul;17(4):e624-32.
PISTELLI GC MARQUES VAS IWAKI LCV

SILVA MC

PIERALISSI N

TOLENTINO ES

CONTRIBUIÇÃO DA RADIOGRAFIA PANORAMICA NO DIAGNÓSTICO DA OSTEOPOROSE
REV. ODONTOL.

UnIV. CID. São

PAULO

2014; 26(1): 71 -

80 , JAN-ABR 
PISTELLI GC

MARQUES VAS

IWAKI LCV

SILVA MC

PIERALISSI N

TOLENTINO ES

CONTRIBUIÇÃO DA RADIOGRAFIA

PANORÂMICA NO

DIAGNÓSTICO DA

OSTEOPOROSE
18. Kavitha MS, Asano A, Taguchi A, Kurita T, Sanada M. Diagnosis of osteoporosis from dental panoramic radiographs using the support vector machine method in a computer-aided system. BMC Medical Imaging [serial on the Internet]. 2012 [Acesso em 31 maio 2013]: Disponível em: http://www. biomedcentral.com/1471-2342/12/1.
19. Shintaku WH, Enciso R, Covington JS, Migliorati CA. Can dental students be taught to use dental radiographs for osteoporosis screening? J Dent Educ 2013 May; 77(5):598-603.

Recebido:25/11/2013

Aceito :02/02/2014 